\title{
Standardization of Ca-45 Radioactive Solution by Tracing Method
}

\author{
Cláudia Regina Ponte Ponge-Ferreira, Marina Fallone Koskinas, and Mauro da Silva Dias \\ Instituto de Pesquisas Energéticas e Nucleares, Caixa Postal 11049, 05422-970, São Paulo, SP, Brazil
}

Received on 8 October, 2003

\begin{abstract}
The procedure followed by the Laboratório de Metrologia Nuclear (LMN) at the IPEN, in São Paulo, for the standardization of the ${ }^{45} \mathrm{Ca}$ is described. The activity measurement was carried out in a $4 \pi \beta-\gamma$ coincidence system, by the tracing method. The radionuclide chosen as the $\beta-\gamma$ emitting tracer nuclide was ${ }^{60} \mathrm{Co}$ because of its end-point beta-ray energy which is close to ${ }^{45} \mathrm{Ca}$. Six sources were prepared using a 1:1 ratio ( $\beta$-pure and $\beta-\gamma$ ) dropped directly on the Collodion film, and other two solutions of ${ }^{45} \mathrm{Ca}+{ }^{60} \mathrm{Co}$ were mixed previously using a 1:1 and 1:2 ratio before making the radioactive sources. The activity of the solution was determined by the extrapolation technique. The events were registered using a Time to Amplitude Converter (TAC) associated with a Multi-channel Analyzer.
\end{abstract}

\section{Introduction}

This paper describes the procedure followed by the Laboratório de Metrologia Nuclear (LMN) at the IPEN CNEN/SP, in São Paulo, for the standardization of ${ }^{45} \mathrm{Ca}$ radioactive solution by tracing method.

This method consists of using $4 \pi \beta-\gamma$ coincidence method [1,2] for the standardization of a pure $\beta$-emitter mixed with another radionuclide which decays by simultaneous emission of two radiations such as $\beta-\gamma, \alpha-\gamma$ to be used as tracer. The tracer is standardized separately by means of conventional $4 \pi \beta-\gamma$ coincidence method.

In the tracing method $[3,4]$ a series of sources containing aliquots of the pure $\beta$-emitter and a suitable $\beta-\gamma$ emitter are prepared. The observed disintegration rate of $\beta$-emitter and the tracer $\beta$-efficiency $\epsilon_{\beta_{t}}$ are measured within a range of $\epsilon_{\beta_{t}}$ by using external absorbers.

The results are plotted against $\left(1-\epsilon_{\beta_{t}}\right)$ and the intercept corresponds to the disintegration rate of the pure $\beta$-emitter.

Radionuclide ${ }^{45} \mathrm{Ca}$ decays with half life of $(163 \pm 1)$ days [8] by beta transition, $0.0017 \%$ populating the excited state of ${ }^{45} \mathrm{Sc}$ and $99.9983 \%$ to the ground state with maximum beta energy of $256 \mathrm{keV}$. Due to the low gamma ray emission probability per decay it may be considered a pure beta emitter radionuclide. ${ }^{45} \mathrm{Ca}$ decay is presented in Fig. 1.

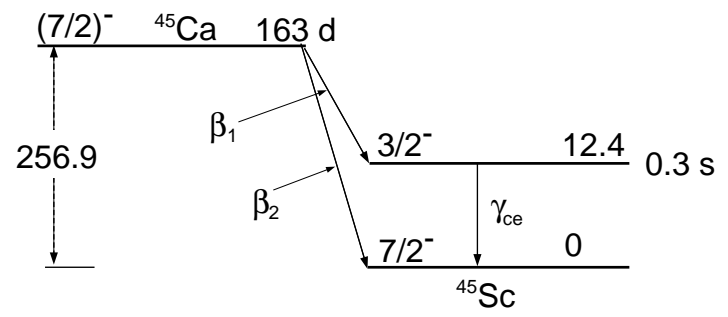

Figure 1. Decay scheme of ${ }^{45} \mathrm{Ca}$. All energies are in $\mathrm{keV}$.
Radionuclide ${ }^{60} \mathrm{Co}$ was chosen as tracer because of its end-point $\beta$-ray energy (317.89 keV) which is close to ${ }^{45} \mathrm{Ca}$. It decays with half-life of $(5.271 \pm 0.002)$ years, by $\beta^{-}$emission populating the excited levels of ${ }^{60} \mathrm{Ni}$ and proceeds to ground state by emission of two main gamma rays (1173.24 and $1332.51 \mathrm{keV})[8]$.

\section{Experimental Method}

\subsection{Source Preparation}

${ }^{45} \mathrm{Ca}$ solution was obtained by means of ${ }^{44} \mathrm{Ca}(\mathrm{n}, \gamma){ }^{45} \mathrm{Ca}$ reaction in a thermal neutron flux at the IPEN $2 \mathrm{MW}$ research reactor. The sources were prepared by dropping known aliquots of the solutions on a $20 \mu \mathrm{g} / \mathrm{cm}^{2}$ thick Collodion film. Six sources were prepared using a $1: 1$ ratio $(\beta$-pure and $\beta-\gamma)$ dropped directly on the Collodion film and other two solutions of ${ }^{45} \mathrm{Ca}+{ }^{60} \mathrm{Co}$ were mixed previously using a 1:1 and 1:2 ratio before making the radioactive sources.

The Collodion film was previously coated with a 10 $\mu \mathrm{g} / \mathrm{cm}^{2}$ gold layer in order to turn the film conductive. A seeding agent (Cyastat SM) was used to improve the deposit uniformity and the sources were dried in a warm (45 degrees Celsius) nitrogen jet. The accurate source mass determination was performed using the picnometer technique.[5] The $\beta-\gamma$ tracer was standardized previously by measuring several sources prepared by the same procedure.

\section{$2.24 \pi \beta-\gamma$ coincidence measurement}

A conventional $4 \pi \beta-\gamma$ coincidence system was used, consisting of a $4 \pi$ proportional counter filled with $0.1 \mathrm{MPa} \mathrm{P}-$ 10 gas mixture, coupled to a pair of 3" x 3" NaI(Tl) crystals. The events were registered by a method developed at LMN which makes use of a Time to Amplitude Converter (TAC) associated with a Multi-channel Analyzer.[7] The gamma 
window was set by gating the gamma-rays of tracer (1173 $\mathrm{keV}+1332 \mathrm{keV}$ ).

The number of detected events in the proportional counter is given by:

$$
N_{\beta(C a+C o)}=N_{0 C o} \epsilon_{\beta C o}+N_{0 C a} \epsilon_{\beta C a}
$$

where:

$\epsilon_{\beta C o}$ is the tracer efficiency in the mixed source;

$N_{0(C a+C o)}$ is the counting rate of proportional counter due to the mixed source;

$N_{0 C o}$ is the activity of ${ }^{60} \mathrm{Co}$ tracer of the mixed source;

$N_{0 C a}$ is the ${ }^{45} \mathrm{Ca}$ beta-branch disintegration rate;

$\epsilon_{\beta C a}$ is the ${ }^{45} \mathrm{Ca}$ beta efficiency.

When the $\beta$-emitter and the $\beta$ - $\gamma$ tracer are combined in a single source, a functional relationship exists between the detection efficiencies. This relation can be defined by a polynomial function $\mathrm{G}$ where:

$$
\left(1-\epsilon_{\beta C a}\right) / \epsilon_{\beta C a}=G\left(\left(1-\epsilon_{\beta C o}\right) / \epsilon_{\beta C o}\right)
$$

Since the tracer efficiency, $\epsilon_{\beta_{C} o}$ may not always be accurately obtainable from coincidence counting data, is convenient to use the expression involving only observed $\beta-\gamma$ and coincidence counting rates.

The expression can be rewritten as:

$$
\begin{gathered}
\frac{N_{\beta(C a+C o)} N_{\gamma C o}}{N_{c C o}}-N_{0 C o} \\
=N_{0 C a}\left[1+G^{\prime}\left(\left(1-\frac{N_{c C o}}{N_{\gamma C o}}\right) / \frac{N_{c C o}}{N_{\gamma C o}}\right)\right]
\end{gathered}
$$

The function $G^{\prime}$ was fitted by weighted least squares using code LINFIT [9] and the extrapolation (1 $\left.N_{c} / N_{\gamma}\right) / N_{c} / N_{\gamma}=0$ gave the expected $N_{0 C a}$ value. Suitable corrections for background, decay, dead time and accidental coincidences were included in calculation.

\section{Results and Discussion}

Figure 2 shows the extrapolation curves obtained for the three different methods of preparing sources: mixing solutions with ratios $1: 1$ and $1: 2$ and by drops with $1: 1$ ratio. The $\beta$ efficiency was varied using external absorbers.

The extrapolated value for the two mixing solutions were in agreement with each other, namely $(154.3 \pm 1.9)$ $\mathrm{kBq} / \mathrm{g}$ and $(154.3 \pm 2.5) \mathrm{kBq} / \mathrm{g}$, respectively. However, for the other preparation method (drops 1:1), the extrapolated value was $(150.3 \pm 1.3) \mathrm{kBq} / \mathrm{g}, 3 \%$ lower. The possible causes for this difference are being investigated.
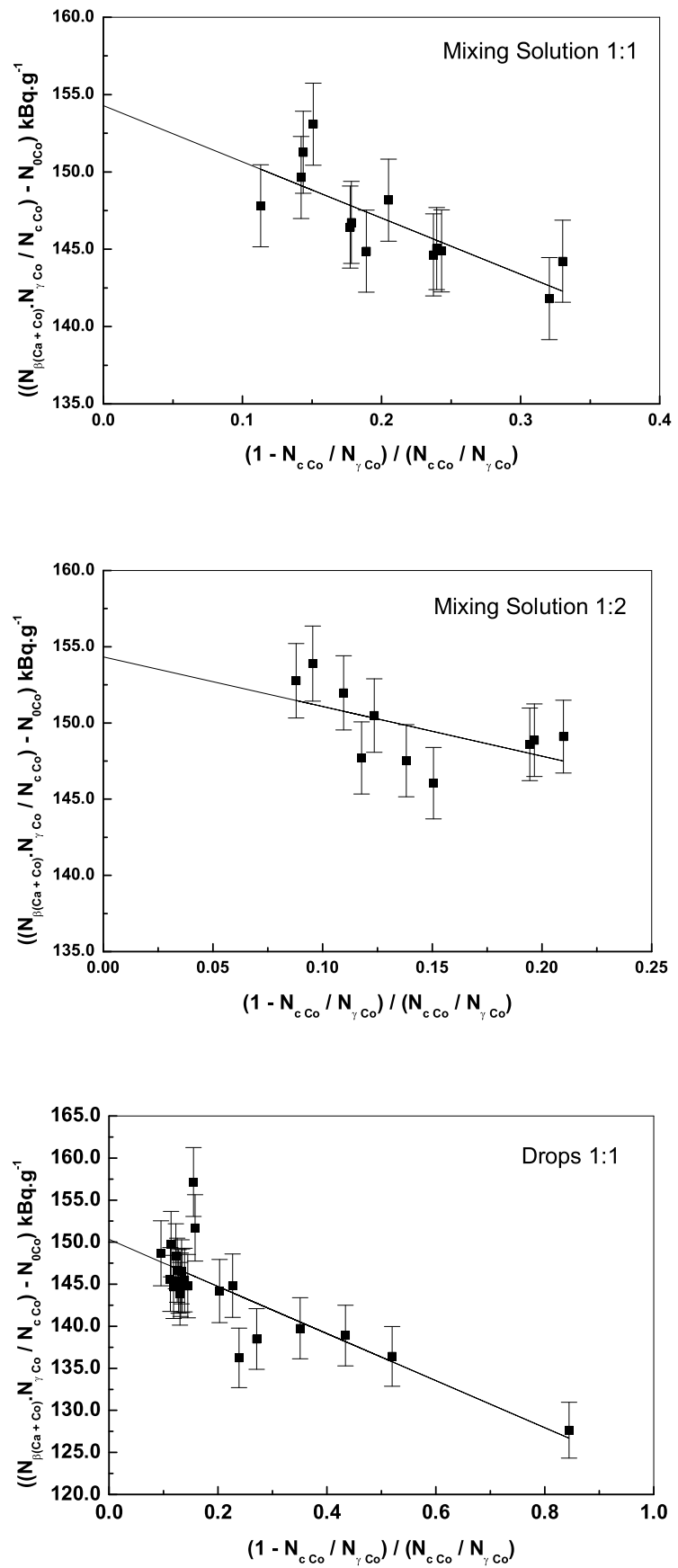

Figure 2. Extrapolation curves of $\frac{N_{\beta(\mathrm{Ca}+\mathrm{Co})} \cdot N_{\gamma \mathrm{Co}}}{N_{c \mathrm{Co}}}-N_{0 \mathrm{Co}}$ as a function of $\frac{1-N_{c C o} / N_{\gamma C o}}{N_{c C o} / N_{\gamma C o}}$.

\section{References}

[1] A. P. Baerg, Metrologia, 3, 105 (1967).

[2] P.J. Campion, Int. J. Appl. Radiat. Iso. 4, 232 (1959).

[3] A. P. Baerg, S. Meghir, and G. C. Bowes, Int. Journ. Appl. Radiat. Isot. 15, 279 (1964).

[4] A. Williams, Int. Journ. Appl. Radiat. Isot. 15, 709 (1964). 
[5] P. J. Campion, Procedures for accurately diluting and dispensing radioactive solutions. Bureau International des Poids et Mesures, Monographie BIPM - 1, 1975

[6] A. M. Baccarelli, M. S. Dias, and M. F. Koskinas, Appl. Radiat. Isot. 58, 239 (2003).

[7] W. O. Lavras, M. F. Koskinas, M. S. Dias, and K. A. Fonseca, Primary Standardization of ${ }^{51} \mathrm{Cr}$ Radioactive Solution. IRPA, 2000 (CDROM).
[8] F. Lagoutine, N. Coursol, and J. Legrand, Table de radionucléides. Laboratoire de Métrologie des Rayonnements Ionisants. Bureau National de Métrologie., 1984.

[9] M. S. Dias, Polynomial least square fitting codes with covariance analysis. Internal Report of the Nuclear Metrology Laboratory, IPEN, 1998 